colonic mucosa in the same patient $(\mathrm{n}=10)$, and from normal controls $(n=10)$. Endoscopic (Baron) and histological assessment was made. An iTRAQ-compatible extraction protocol for insoluble IF proteins was developed. Labelled peptides from pooled patients were analysed by SCX-LC-MS/MS (strong cation exchange-reverse phase HPLC tandem mass spectrometry) and data reconstituted in GeneBio Phenyx. Inter-group comparisons were made using in-house algorithms based on t-testing with multiple test correction.

Results Median age was 36 years (range 23-71). Endoscopic Baron score was $\geq 2$ in inflamed mucosa for all patients. Tandem mass spectrometry (MS/MS) identified 52 proteins, 32 (61.5\%) matched by two or more peptides, showing significant log fold change, with reduced levels of keratins and vimentin in inflamed mucosa compared to controls (Abstract PMO-250 table 1). Abstract PMO250 figure 1 shows significantly reduced IF protein levels in inflamed mucosa compared to inactive mucosa. Cytokine proteins neutrophil defensin 1 and bone morphogenetic protein 4 were increased only in actively inflamed mucosa.

Abstract PM0-250 Table 1 Inflamed and non-inflamed mucosa vs controls (log fold change)

\begin{tabular}{llllll}
\hline Protein & Accession no & MolWeight (kDa) & Peptide no & Active & Inactive \\
\hline K8 & P05787 & 53.7 & 35 & $0.4^{*}$ & NS \\
K18 & P05783 & 48.0 & 17 & $0.7^{*}$ & NS \\
K19 & P08727 & 44.1 & 31 & $0.4^{*}$ & NS \\
Vimentin & B3KRK8 & 46.9 & 4 & $0.6^{*}$ & NS \\
\hline
\end{tabular}

${ }^{*} \mathrm{p}<0.05$.

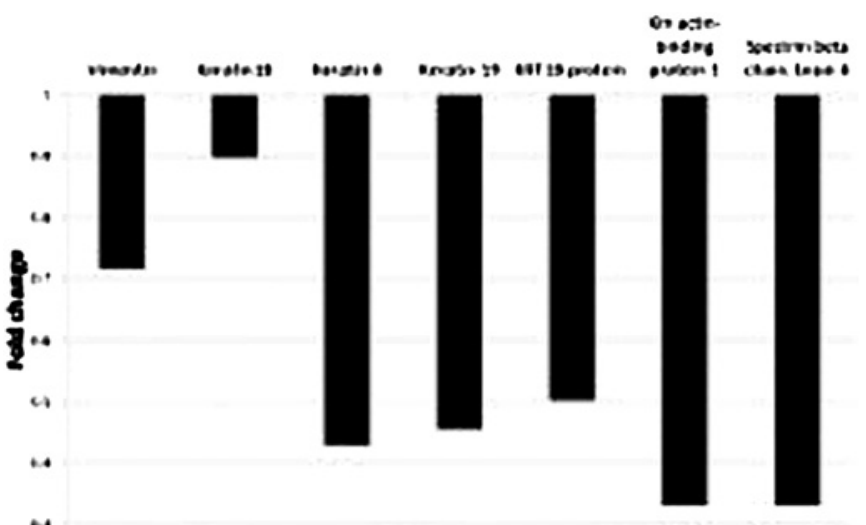

Abstract PM0-250 Figure 1 Decreased levels of IF proteins in inflamed colonic mucosa.

Conclusion Using a quantitative proteomic approach, we have shown significantly decreased levels of keratins in the actively inflamed colonic mucosa in UC providing further evidence of interaction between keratins and inflammatory pathways-which requires further elucidation.

Competing interests None declared.

\section{PM0-251 A RETROSPECTIVE AUDIT OF COLORECTAL CANCER SURVEILLANCE IN INFLAMMATORY BOWEL DISEASE IN SECONDARY CARE}

doi:10.1136/gutjnl-2012-302514b.251

K Burley, E Arthurs, ${ }^{*}$ B Gholkar, L Williams, M Lockett. Gastroenterology, North Bristol NHS Trust, Bristol, UK

Introduction Patients with colitis are at increased risk of colorectal cancer (CRC). Colonoscopic surveillance to detect dysplasia and early cancers has been advocated by the BSG since $2002{ }^{1}$ Our aims were to assess whether patients with colitis in our patient cohort are receiving appropriate colonoscopic surveillance for CRC according to these guidelines, and to assess the impact of the updated 2010 BSG guidelines ${ }^{2}$ on local endoscopy services.

Methods Patients with IBD were identified from secondary care coding databases and verified by paper records. A retrospective review of case notes was performed. Data on diagnoses, duration of symptoms, extent of disease and CRC surveillance was collected and analysed. Individualised recommendations for colonoscopic screening and surveillance were made according to the 2010 BSG guidelines.

Results 45 colitis patient records were reviewed; 20 CD: 25 UC, M:F 23:22. The average age was 59.4 (range 18.6-87); average duration of disease 18.6 (range $0-56) .35$ (78\%) had colitis extent requiring surveillance. 26 patients $(58 \%)$ had symptom onset $>10$ years; 11 patients (42\%) underwent screening colonoscopy at 8-10 years; 14 $(54 \%)$ did not, one patient underwent colonoscopy but date of diagnosis was unclear. Nine patients $(35 \%)$ underwent inconsistent surveillance, in six patients $(23 \%)$ there was no record of a colonoscopy. Reasons for inconsistent or absent surveillance included nonattendance (2), patient declined (1) and unclear (11). 24 patients were eligible for repeat colonoscopy; 3 (13\%) underwent this at the recommended interval; one patient was due in 2011; $11(46 \%)$ underwent inconsistent surveillance; nine (38\%) did not undergo any surveillance. Reasons for absent or inconsistent surveillance included non-attendance (2), lost to follow-up (1), patient declined (1), procedure unnecessary due to disease extent (1), patient undergoing surgery in the interval between colonoscopies (1) and unclear (14). Of 26 patients eligible for surveillance, 3 were excluded due to disease extent and intervening surgery. Of 23 remaining patients, the surveillance interval between colonoscopies would be increased in 12 patients (52\%), unchanged in $6(26 \%)$ and reduced in $3(13 \%)$ with the introduction of the 2010 BSG guidelines. The impact was unclear in two patients (9\%).

Conclusion Patients with colitis in our patient cohort at NBT are not receiving appropriate CRC surveillance according to BSG guidelines. These results emphasise the need for a robust coordinated surveillance programme. The 2010 guidelines have had the net effect of increasing the time interval between colonoscopies, which may lead to an overall reduction in endoscopy workload from surveillance cases.

Competing interests None declared.

\section{REFERENCES}

1. Eaden JA, Mayberry JF. Gut 2002;51(Suppl V):v10-12

2. Cairns SR, et al. Guidelines. Gut 2010;59:666-90.

\section{PMO-252 EFFICACY OF INFLIXIMAB THERAPY IN ACUTE AND SUB- ACUTE ULCERATIVE COLITIS}

doi:10.1136/gutjnl-2012-302514b.252

E Harrison,* J Slater, L Wood, S C Cooper, S de Silva. Department of Gastroenterology, Dudley Group of Hospitals NHS Foundation Trust, Birmingham, UK

Introduction Ulcerative colitis (UC) has a chronic relapsing course. Infliximab is beneficial in severe disease, but conflicting data exists regarding the subsequent colectomy rates. We aimed to review outcomes post-infliximab usage in acute and sub-acute UC in our clinical practice.

Methods We conducted a retrospective review of all patients who had received a maximum of three doses of infliximab for acute or sub-acute exacerbations of UC between January 2010 and October 2011. Medical treatment (initial and subsequent) and colectomy rates were recorded for all patients. 